\title{
Association of serum bone- and muscle- derived factors with age, sex, body composition, and physical function in community-dwelling middle-aged and elderly adults: a cross-sectional study
}

Kenta Moriwaki ${ }^{1 *}$, Hiromi Matsumoto ${ }^{2}$, Shinji Tanishima ${ }^{1}$, Chika Tanimura ${ }^{3}$, Mari Osaki ${ }^{4}$, Hideki Nagashima ${ }^{1}$ and Hiroshi Hagino $0^{3,4}$

\begin{abstract}
Background: Understanding interactions between bone and muscle based on endocrine factors may help elucidate the relationship between osteoporosis and sarcopenia. However, whether the abundance or activity of these endocrine factors is affected by age and sex or whether these factors play a causal role in bone and muscle formation and function is unclear. We aimed to evaluate the association of serum bone- and muscle-derived factors with age, sex, body composition, and physical function in community-dwelling middle-aged and elderly adults.

Methods: In all, 254 residents (97 men, 157 women) participated in this cross-sectional study conducted in Japan. The calcaneal speed of sound (SOS) was evaluated by quantitative ultrasound examination. Skeletal muscle mass index (SMI) was calculated by bioelectrical impedance analysis. Grip strength was measured using a dynamometer. Gait speed was measured by optical-sensitive gait analysis. Serum sclerostin, osteocalcin (OC), insulin-like growth factor-1 (IGF-1), myostatin, and tartrate-resistant acid phosphatase-5b (TRACP-5b) concentrations were measured simultaneously. The difference by sex was determined using $t$ test. Correlations between serum bone- and musclederived factors and age, BMI, SOS, SMI, grip strength, gait speed, and TRACP-5b in men and women were determined based on Pearson's correlation coefficients. Multiple regression analysis was performed using the stepwise method.

Results: There was no significant difference with regard to age between men (75.0 \pm 8.9 years) and women (73.6 \pm 8.1 years). Sclerostin was significantly higher in men than in women and tended to increase with age in men; it was significantly associated with SOS and TRACP-5b levels. OC was significantly higher in women than in men and was significantly associated with TRACP-5b levels and age. IGF-1 tended to decrease with age in both sexes and was significantly associated with SOS and body mass index. Myostatin did not correlate with any assessed variables.

Conclusions: Sclerostin was significantly associated with sex, age, and bone metabolism, although there was no discernable relationship between serum sclerostin levels and muscle function. There was no obvious relationship between OC and muscle parameters. This study suggests that IGF-1 is an important modulator of muscle mass and function and bone metabolism in community-dwelling middle-aged and elderly adults.
\end{abstract}

Keywords: Sclerostin, Insulin-like growth factor-1, Osteocalcin, Myostatin, Osteoporosis, Sarcopenia

\footnotetext{
* Correspondence: moriwakik5@gmail.com

${ }^{1}$ Department of Orthopedic Surgery, Faculty of Medicine, Tottori University,

Nishicho 36-1, Yonago, Tottori 683-8504, Japan

Full list of author information is available at the end of the article
}

(c) The Author(s). 2019 Open Access This article is distributed under the terms of the Creative Commons Attribution 4.0 International License (http://creativecommons.org/licenses/by/4.0/), which permits unrestricted use, distribution, and reproduction in any medium, provided you give appropriate credit to the original author(s) and the source, provide a link to the Creative Commons license, and indicate if changes were made. The Creative Commons Public Domain Dedication waiver (http://creativecommons.org/publicdomain/zero/1.0/) applies to the data made available in this article, unless otherwise stated. 


\section{Background}

Osteoporosis and sarcopenia are global problems that increase with longer life expectancy. Osteoporosis elevates the risk of fragility fractures and poor quality of life, and imposes a heavy economic burden [1]. Sarcopenia is characterized by a decline in muscle strength and mobility and an increase in falls and disability [2]. The prevalence of sarcopenia in Japanese women with osteoporosis was $29.7 \%$ in 2013 [3]. The risk of low bone mineral density (BMD) of the femoral neck and lumbar spine has been shown to increase significantly with sarcopenia in the Taiwanese population [4]. Therefore, understanding the interactions between bone and muscle may help elucidate the relationship between osteoporosis and sarcopenia.

Muscles interact with bones mechanically and functionally in skeletal tissues. Endocrine factors, as well as mechanical factors, may affect both muscle and bone metabolism. Sclerostin, a bone-derived factor, is an important negative regulator of bone formation and plays a key role in regulating the response to mechanical loading $[5,6]$. Several previous studies have shown that men have significantly higher serum sclerostin concentrations than women and that serum sclerostin concentrations are positively correlated with age in healthy men $[7,8]$. Serum sclerostin was positively correlated with BMD in the lumbar vertebrae and femur, as measured by dual energy Xray absorptiometry (DXA), in postmenopausal women and with trabecular volumetric BMD, as measured by high-resolution peripheral quantitative computed tomography, in adult men $[8,9]$. Sclerostin is an important negative regulator of bone formation that plays a key role in regulating the response to mechanical loading $[5,6]$. Serum sclerostin was significantly lower in a group with high levels of physical activity than in a group with low levels of physical activity [7], whereas sclerostin was higher in patients with paralysis resulting from spinal cord injuries than in those with no injury [10].

Osteocalcin (OC), secreted by osteoblasts, is often used as a marker of bone formation [11]. A previous study demonstrated the relationship between bone metabolism markers and mechanical loading. OC and Insulin-like growth factor-1 (IGF-1) were significantly positively correlated with high-force eccentric exercise, and exercise caused an increase in $O C$ and serum tartrate-resistant acid phosphatase-5b (TRACP-5b), which promotes increased bone metabolism [12].

IGF-1 is known for its physiological and pathological roles in the regulation of bone metabolism [13, 14]. Several studies suggest that IGF-1 is an important modulator of muscle mass and function, not only during the developmental period but also across the entire life span [15]. Serum IGF-1 concentrations are significantly lower in elderly women than in young women [16]. High serum IGF-1 concentrations are associated with the likelihood of being sarcopenic [17].

Myostatin is a highly conserved member of the transforming growth factor- $\beta$ family functioning as a potent negative regulator of growth, and it is highly concentrated in skeletal muscles. Since the discovery of myostatin in skeletal muscles, there has been great interest in its role as a potential mediator of sarcopenia and as a therapeutic target [18]. Regarding serum myostatin levels, several reports have indicated different results; myostatin was reported to be significantly higher in men than in women and it negatively correlated with lean body and muscle mass $[19,20]$. In contrast, in another study, myostatin was reported to positively correlate with muscle mass [21]. Further, age-related declines in bone mineral content and density were reported to be attenuated in myostatin-deficient mice [22].

Understanding the interactions between bone and muscle based on endocrine factors may help elucidate the relationship between osteoporosis and sarcopenia. However, it is unclear whether the abundance or activity of these endocrine factors is affected by age and sex or whether these factors play a causal role in bone and muscle metabolism. Thus, this study aimed to evaluate the association of serum bone- and muscle-derived factors with age, sex, body composition, and physical function in community-dwelling middle-aged and elderly adults.

\section{Methods \\ Participants}

This is a cross-sectional, population-based, observational study conducted in the town of Hino, Tottori Prefecture, Japan [2, 23-25]. In 2015, the town population comprised 3278 residents and approximately $47 \%$ of the residents were 65 years or older. Study participants were recruited from a pool of individuals who had registered for an annual town-sponsored medical check-up. A total of 1357 individuals aged 40 years or older were eligible to receive the annual town-sponsored medical check-up in 2016. Three individuals were excluded because it was certified that they required long-term care. A selfadministered questionnaire was sent to 1354 participants. The inclusion criteria for participation in the study were as follows: (i) agreement to participate; (ii) living independently; and (iii) the ability to walk to the survey site and provide self-reported data. Accordingly, a total of 254 residents (97 men, 157 women) participated in the study.

\section{Characteristics of participants}

Characteristics of participants such as age, sex, height, weight, and body mass index (BMI) were recorded during the medical check-up. 


\section{Quantitative bone ultrasound imaging}

Quantitative ultrasound (QUS) imaging was used to assess calcaneal bone mass. The measured calcaneal speed of sound (SOS) was evaluated using an ultrasound bone densitometer (CM-200; Furuno Electric Co., Nishinomiya, Japan) with a temperature correcting function. All participants placed their right heel on the quantitative ultrasound device while being seated. A coupling gel was applied to each participant's right heel to facilitate the transmission of ultrasound waves to the skeletal site being examined.

\section{Muscle mass}

Muscle mass was measured by bioelectrical impedance analysis (BIA) using a body composition analyzer (MC-780A; Tanita Co., Tokyo, Japan). The BIA method required participants to step onto a platform resembling a bathroom scale and remain in a standing position for approximately $30 \mathrm{~s}$. Skeletal muscle mass index (SMI) was calculated by dividing limb muscle mass by height $\left(\mathrm{kg} / \mathrm{m}^{2}\right)$.

\section{Grip strength}

Grip strength was measured using a dynamometer (T.K.K.5401; Takei Scientific Instruments Co., Niigata, Japan). Each participant was asked to squeeze the dynamometer twice with each hand. The highest score for each hand was recorded as the representative value.

\section{Gait speed}

Gait speed was measured once for each participant by optical-sensitive gait analysis (Optogait; Microgate Co., Bolzano, Italy). Each participant was instructed to walk at their normal speed and the mean gait speed was calculated using a software program (Optogait analysis software, version 1.6.4.0; Microgate Co.) during $5 \mathrm{~m}$ of walking at a comfortable speed.

\section{Serum biochemical measurements}

Blood samples were collected at the same time when parameters of body structure and physical function were measured. Samples were centrifuged for $15 \mathrm{~min}$ at 3000 r.p.m. at $4{ }^{\circ} \mathrm{C}$ and stored at $-80^{\circ} \mathrm{C}$ until final analysis. Serum sclerostin levels were measured using an enzymelinked immuno-sorbent assay (ELISA) kit (Sclerostin ELISA kit; BioMedica, Vienna, Austria). Assay buffer $(150 \mu \mathrm{L} /$ well) was added to $20 \mu \mathrm{L}$ of standards, controls, and samples; this was followed by the addition of $50 \mu \mathrm{L}$ of sclerostin antibody to each well. The plate was incubated for $24 \mathrm{~h}$ at $24{ }^{\circ} \mathrm{C}$. Then, wells were washed five times, and $200 \mu \mathrm{L}$ of conjugate was added. The plate was incubated in the dark for $1 \mathrm{~h}$. Wells were washed, and

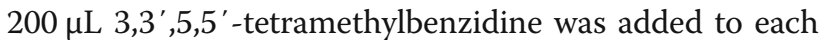
well. Color was allowed to develop for $30 \mathrm{~min}$ at $24^{\circ} \mathrm{C}$, followed by the addition of $50 \mu \mathrm{L}$ of stop solution. Absorbance was read at $450 \mathrm{~nm}$ within $10 \mathrm{~min}$. Serum myostatin levels were measured using an ELISA kit (Myostatin ELISA kit; Immundiagnostik, Bensheim, Germany). Serum IGF-1 concentrations were measured by radioimmunoassay. Serum OC concentrations were measured by an electrochemiluminescence immunoassay. Serum TRACP-5b concentrations were measured by an enzyme immunoassay.

\section{Statistical analysis}

The differences in age, height, weight, BMI, SOS, SMI, grip strength, gait speed, and sclerostin, OC, IGF-1, myostatin, and TRACP-5b levels between sexes were determined using the Student's t test. Associations between serum bone and muscle-derived factors and age in men and women were determined based on Pearson's correlation coefficients. Correlations between serum bone and muscle-derived factors and age, sex, BMI, SOS, SMI, grip strength, gait speed, and TRACP-5b levels were determined based on Pearson's correlation coefficients. Multiple regression analysis was performed with serum sclerostin, OC, and IGF-1 as dependent variables and age, sex, BMI, SOS, SMI, grip strength, and gait speed as independent variables. Selection of regression models was done using the stepwise method. We judged multicollinearity by variance inflation factor (VIF). All data were analyzed using SPSS statistical software (version 24 for Windows; IBM Co., Tokyo, Japan). A $p$-value $<0.05$ was considered significant.

\section{Results}

Demographics, parameters of body composition and physical function, and serum bone and muscle-derived factors

Demographics, parameters of body composition and physical function, and serum bone and muscle-derived factors for men and women and the results of the statistical comparison between them are shown in Table 1. There was no significant difference between men (75.0 \pm 8.9 years) and women $(73.6 \pm 8.1$ years $)$ with regard to mean age $(p=0.198)$. BMI was significantly higher in men than in women $(p=0.014)$. Right calcaneal SOS, SMI, and grip strength were significantly higher in men than in women $(p<0.001)$. Sclerostin levels were significantly higher in men than in women $(p<0.001)$. OC concentration was significantly higher in women than in men $(p<0.001)$. TRACP-5b levels were significantly higher in women than in men $(p=0.026)$.

\section{Association of serum bone and muscle-derived factors with age between sexes}

Serum sclerostin and $\mathrm{OC}$ concentrations are shown by scatter plots in Fig. 1. Sclerostin was positively 
Table 1 Demographics, parameters of body composition and physical function, and serum bone- and muscle-derived factors

\begin{tabular}{|c|c|c|c|}
\hline & Men $(n=97)$ & Women $(n=157)$ & $p^{a}$ \\
\hline Age (years) & $75.0 \pm 8.9$ & $73.6 \pm 8.1$ & 0.198 \\
\hline Height (cm) & $163.1 \pm 6.5$ & $150.0 \pm 6.5$ & $<0.001$ \\
\hline Weight (kg) & $60.9 \pm 8.0$ & $49.5 \pm 8.0$ & $<0.001$ \\
\hline BMI $\left(\mathrm{kg} / \mathrm{m}^{2}\right)$ & $23.0 \pm 2.5$ & $22.0 \pm 3.1$ & 0.014 \\
\hline $\operatorname{SOS}^{b}(\mathrm{~m} / \mathrm{s})$ & $1503.6 \pm 28.3$ & $1483.4 \pm 20.2$ & $<0.001$ \\
\hline SMI $\left(\mathrm{kg} / \mathrm{m}^{2}\right)$ & $7.5 \pm 0.8$ & $6.1 \pm 0.8$ & $<0.001$ \\
\hline Grip strength (kg) & $34.9 \pm 7.4$ & $23.0 \pm 4.5$ & $<0.001$ \\
\hline Gait speed (m/s) & $1.2 \pm 0.3$ & $1.3 \pm 0.3$ & 0.330 \\
\hline Sclerostin (pmol/L) & $55.9 \pm 26.1$ & $32.6 \pm 13.8$ & $<0.001$ \\
\hline OC (ng/ml) & $16.6 \pm 5.5$ & $21.5 \pm 8.9$ & $<0.001$ \\
\hline IGF-1 (ng/ml) & $97.1 \pm 30.5$ & $93.4 \pm 29.8$ & 0.350 \\
\hline Myostatin (ng/ml) & $42.9 \pm 10.3$ & $43.9 \pm 12.2$ & 0.495 \\
\hline TRACP-5b (mU/dL) & $289.9 \pm 95.4$ & $340.3 \pm 147.4$ & 0.026 \\
\hline
\end{tabular}

$B M I$, body mass index; SMI, skeletal muscle mass index; SOS, speed of sound; OC, osteocalcin; IGF-1, insulin-like growth factor-1;TRACP-5b, tartrate-resistant acid phosphatase-5b

All value are means \pm SD

${ }^{\text {a }}$ Difference between men and women ( $t$ test)

${ }^{\mathrm{b}} \mathrm{SOS}$ of right calcaneus correlated with age in men $(r=0.318, p=0.002)$ and women $(\mathrm{r}=0.180, p=0.024)$. OC was positively correlated with age in women $(\mathrm{r}=0.314, \mathrm{p}=0.002)$ but not in men $(r=-0.075, p=0.358)$. Serum IGF-1 and myostatin concentrations are shown in Fig. 2. IGF-1 was negatively correlated with age in men $(r=-0.290, p<0.001)$ and women $(r=-0.296, p=0.003)$. Serum myostatin was not correlated with age in both men $(r=0.058, p=0.474)$ and women $(\mathrm{r}=-0.029, p=0.781)$.

\section{Correlations of serum bone and muscle-derived factors with demographics and parameters of body composition and physical function}

Pearson's correlation coefficients are shown in Table 2. Sclerostin was positively correlated with age, height, weight, BMI, SOS, SMI, and grip strength, and negatively correlated with sex, OC, TRACP-5b. OC was positively correlated with sex and TRACP-5b, and negatively correlated with height, weight, SOS, SMI, and grip strength. IGF-1 was positively correlated with height, weight, BMI, SOS, SMI, grip strength, and gait speed, and negatively correlated with age, OC, and TRACP-5b. Myostatin was not correlated with any of the assessed variables.

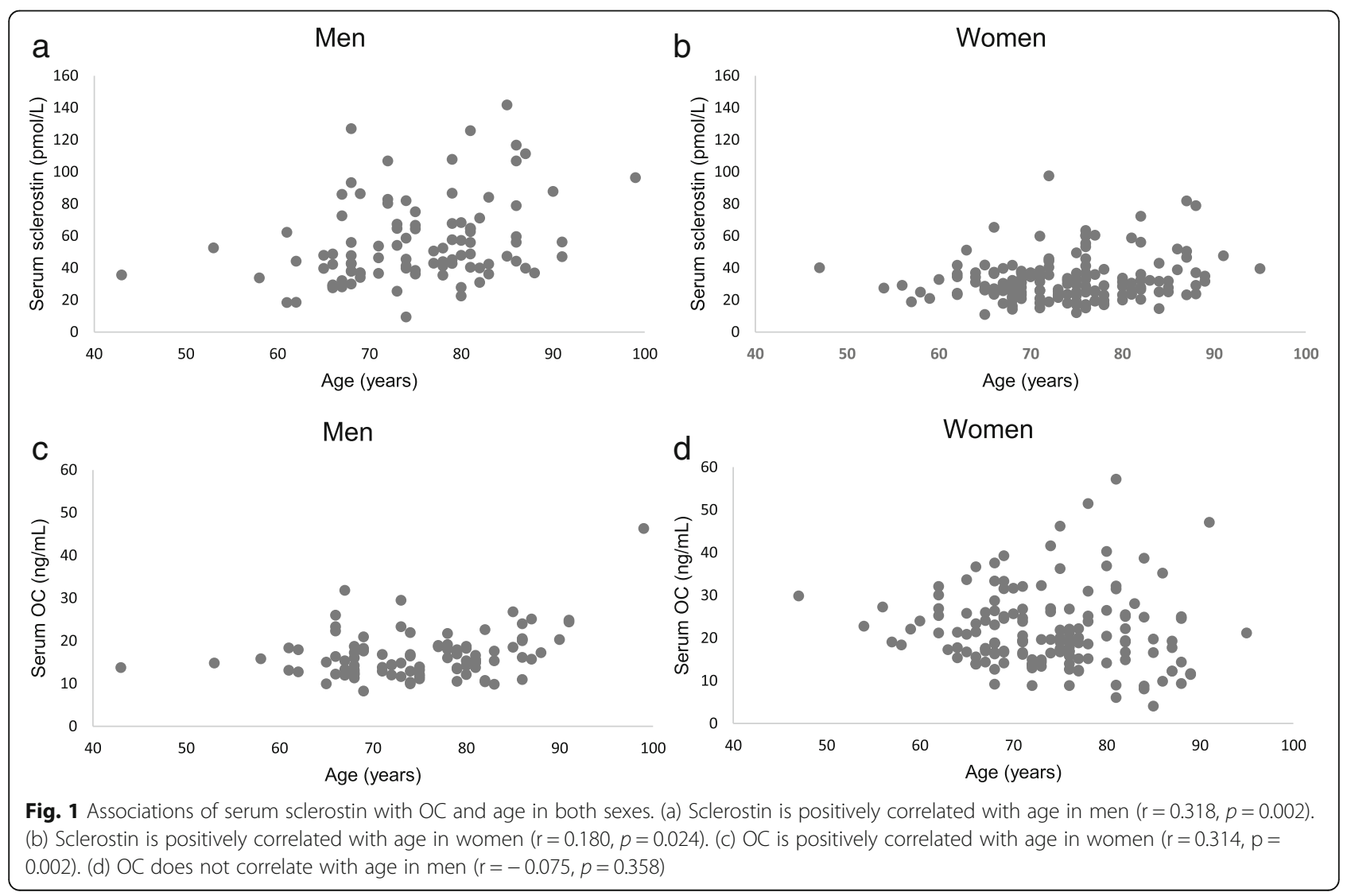



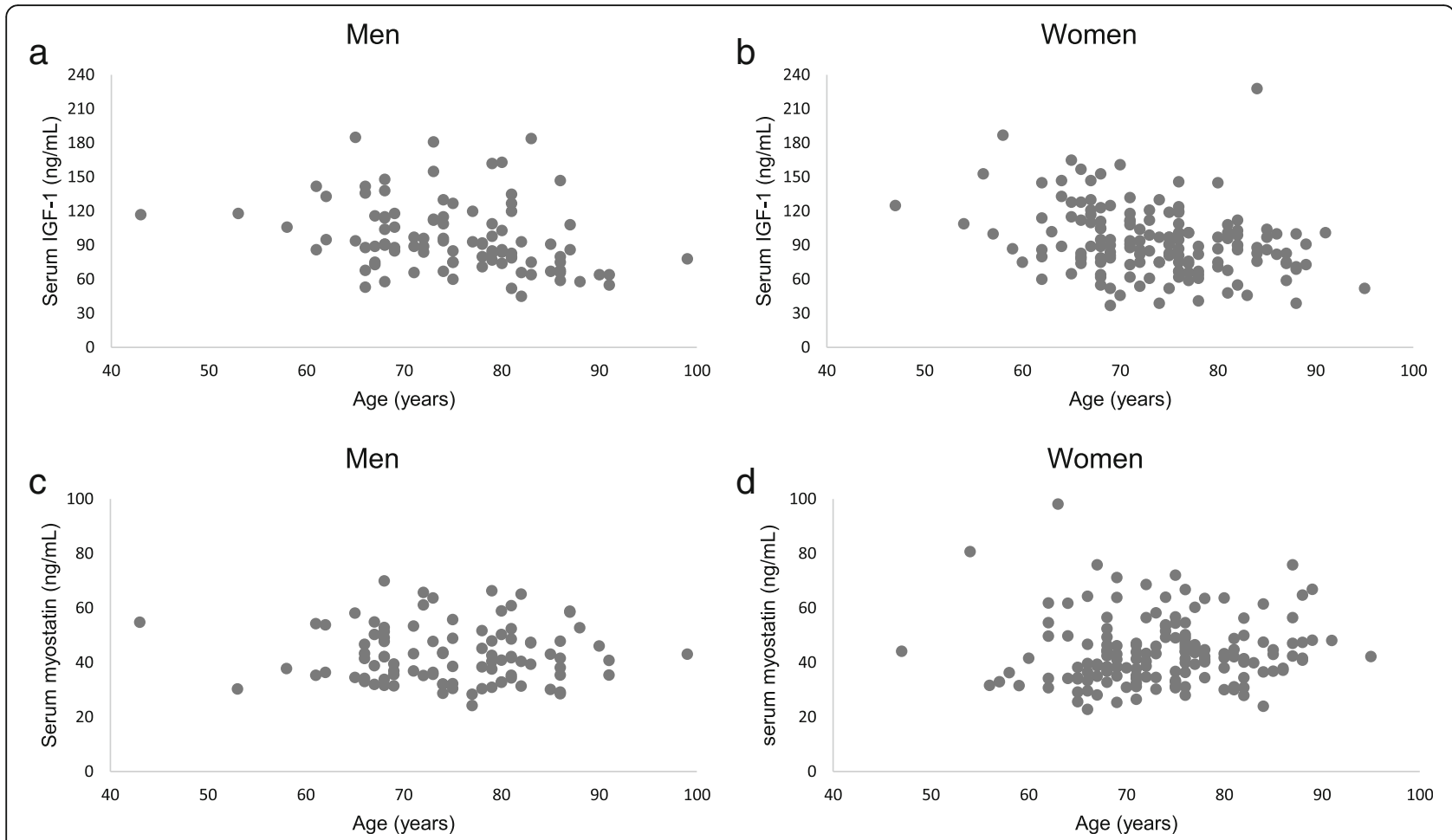

Fig. 2 Associations of serum IGF-1 and myostatin with age in both sexes. (a) IGF-1 is negatively correlated with age in men ( $r=-0.290, p<$ 0.001). (b) IGF-1 is negatively correlated with age in women ( $r=-0.296, p=0.003)$. (c) Myostatin does not correlate with age in men ( $r=0.058$, $p=0.474)$. (d) Myostatin does not correlate with age in women $(r=-0.029, p=0.781)$

\section{Multiple regression analysis}

Multiple regression analysis results are shown in Table 3. Independent predictors for sclerostin were sex (partial regression coefficient $[\mathrm{B}]=18.91$, standardized partial regression coefficient $[\beta]=0.40, p<0.001)$, age $(B=0.75, \beta=0.28$, $p<0.001)$, SOS $(\mathrm{B}=0.14, \beta=0.15, p=0.009)$, and TRACP$5 \mathrm{~b}(\mathrm{~B}=-0.02, \beta=-0.11, p=0.037)$. Independent predictors for osteocalcin were TRACP-5b $(B=0.03, \beta=0.62$, $p<0.001)$, sex $(\mathrm{B}=-2.49, \beta=-0.15, p=0.002)$, and age $(\mathrm{B}=-0.10, \beta=-0.11, p=0.026)$. Independent predictors for IGF-1 were age $(B=-0.86, \beta=-0.24, p<0.001)$, SOS $(\mathrm{B}=0.19, \beta=0.16, p=0.010)$, and $\mathrm{BMI}(\mathrm{B}=1.58, \beta=0.14$, $p=0.018)$. There was no independent predictor for myostatin.

\section{Discussion}

In this study, we evaluated the association of sclerostin, OC, IGF-1, and myostatin with age, sex, BMI, SOS (as a measure of bone mass), SMI (as a measure of muscle mass), grip strength (as a measure of muscle strength), gait speed (as a measure of physical function), and TRACP-5b (as an indicator of bone metabolism).

Sclerostin concentrations were significantly higher in men, and SOS was an independent positive predictor of sclerostin. Although this result is paradoxical given that sclerostin is an inhibitor of bone formation, one possible explanation for this positive correlation between sclerostin and SOS is that bones of a high density are rich in osteocytes, which produce sclerostin [26].

Sclerostin is an important negative regulator of bone formation that plays a key role in regulating the response to mechanical loading $[5,6]$. Immobilized patients have higher serum sclerostin concentrations, associated with reduced bone formation [27]. These findings may be related to the mechanical effects muscles have on bones. During this study, we investigated the relationship between sclerostin and muscle and physical function, and determined that there was no observable obvious relationship between them. This may be because of the difference between immobilized patients and healthy adults. The subjects included in this study were community-dwelling adults with fairly higher activities than immobilized patients. The subjects had sufficient mechanical loading to their bones prior to enrollment in the study, which may have resulted in a decline in the relationship between serum sclerostin concentrations and physical function.

In postmenopausal women immobilized after a stroke, sclerostin correlated negatively with bone formation markers and positively with resorption markers [27]. The results of bone turnover markers suggest that there exists an imbalance between bone resorption and bone 


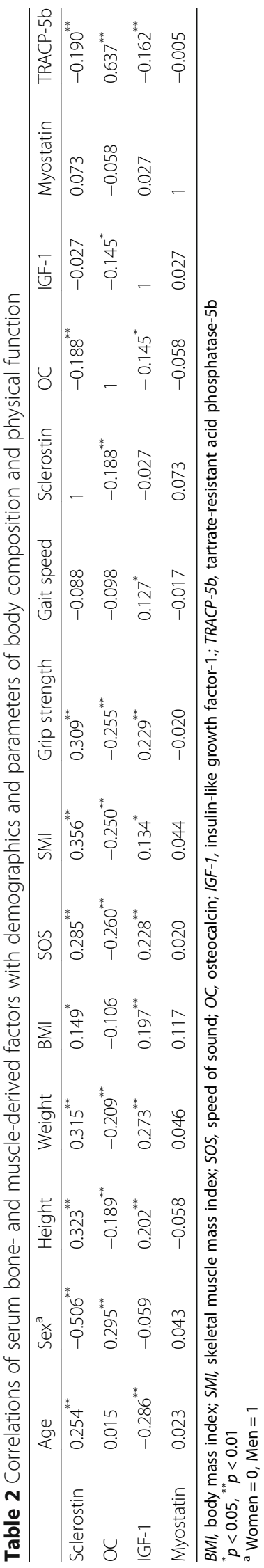


Table 3 Multiple regression analysis

\begin{tabular}{|c|c|c|c|c|c|c|c|c|}
\hline model & & B & $\mathrm{SE}(\mathrm{B})$ & $\beta$ & $\mathrm{t}$ & $95 \% \mathrm{Cl}$ & $p$ & $R^{2}$ \\
\hline \multicolumn{9}{|c|}{ (a) Sclerostin as dependent variable } \\
\hline 1 & Sex $x^{a}$ & 24.14 & 2.56 & 0.52 & 9.40 & (19.08 29.20) & $<0.001$ & 0.267 \\
\hline \multirow[t]{2}{*}{2} & $\operatorname{Sex}^{a}$ & 23.13 & 2.50 & 0.49 & 9.24 & $(18.20 \sim 28.06)$ & $<0.001$ & 0.311 \\
\hline & Age & 0.58 & 0.14 & 0.21 & 4.04 & $(0.30 \sim 0.86)$ & $<0.001$ & \\
\hline \multirow[t]{3}{*}{3} & $\operatorname{Sex}^{a}$ & 19.71 & 2.72 & 0.42 & 7.23 & (14.34 25.08) & $<0.001$ & 0.332 \\
\hline & Age & 0.71 & 0.14 & 0.26 & 4.80 & $(0.42 \sim 1.01)$ & $<0.001$ & \\
\hline & SOS & 0.15 & 0.05 & 0.17 & 2.92 & $(0.50 \sim 0.25)$ & 0.004 & \\
\hline \multirow[t]{4}{*}{4} & $\operatorname{Sex}^{\mathrm{a}}$ & 18.91 & 2.73 & 0.40 & 6.91 & (13.52 24.29) & $<0.001$ & 0.342 \\
\hline & Age & 0.75 & 0.14 & 0.28 & 5.06 & $(0.46 \sim 1.05)$ & $<0.001$ & \\
\hline & SOS & 0.14 & 0.05 & 0.15 & 2.64 & $(0.03 \sim 0.24)$ & 0.009 & \\
\hline & TRACP5b & -0.02 & 0.01 & -0.11 & -2.10 & $(-0.03 \sim-0.001)$ & 0.037 & \\
\hline \multicolumn{9}{|c|}{ (b) OC as dependent variable } \\
\hline 1 & TRACP-5b & 0.03 & 0.003 & 0.63 & 12.78 & $(0.03 \sim 0.04)$ & $<0.001$ & 0.404 \\
\hline \multirow[t]{2}{*}{2} & TRACP-5b & 0.03 & 0.003 & 0.60 & 12.15 & $(0.03 \sim 0.04)$ & $<0.001$ & 0.428 \\
\hline & Sex $x^{a}$ & -2.73 & 0.81 & -0.16 & -3.36 & $(-4.33 \sim-1.13)$ & 0.001 & \\
\hline \multirow[t]{3}{*}{3} & TRACP-5b & 0.03 & 0.003 & 0.62 & 12.46 & $(0.03 \sim 0.04)$ & $<0.001$ & 0.438 \\
\hline & $\operatorname{Sex}{ }^{a}$ & -2.49 & 0.81 & -0.15 & -3.06 & $(-4.09 \sim-0.89)$ & 0.002 & \\
\hline & Age & -0.10 & 0.04 & -0.11 & -2.23 & $(-0.19 \sim-0.01)$ & 0.026 & \\
\hline \multicolumn{9}{|c|}{ (c) IGF-1 as dependent variable } \\
\hline 1 & Age & -1.03 & 0.22 & -0.29 & -4.68 & $(-1.47 \sim-0.60)$ & $<0.001$ & 0.080 \\
\hline \multirow[t]{2}{*}{2} & Age & -0.88 & 0.22 & -0.24 & -3.93 & $(-1.32 \sim-0.42)$ & $<0.001$ & 0.107 \\
\hline & SOS & 0.21 & 0.07 & 0.17 & 2.85 & $(0.06 \sim 0.35)$ & 0.005 & \\
\hline \multirow[t]{3}{*}{3} & Age & -0.86 & 0.22 & -0.24 & -3.87 & $(-1.30 \sim-0.42)$ & $<0.001$ & 0.124 \\
\hline & SOS & 0.19 & 0.07 & 0.16 & 2.60 & $(0.04 \sim 0.33)$ & 0.010 & \\
\hline & BMl & 1.58 & 0.66 & 0.14 & 2.38 & $(0.27 \sim 2.89)$ & 0.018 & \\
\hline
\end{tabular}

$B$, partial regression coefficient; $S E$, standard error; $\beta$, standardized partial regression coefficient; $t$, t-ratio; $95 \% C l$, $95 \%$ confidence interval; $p, p$-value; $R^{2}$, coefficient of determination

$B M I$, body mass index; IGF-1, insulin-like growth factor-1; OC, osteocalcin; SMI, skeletal muscle mass index; SOS, speed of sound; TRACP-5b, tartrate-resistant acid phosphatase-5b

Multiple regression analysis was performed with serum sclerostin, OC, and IGF-1 as dependent variables, and with age, gender, BMI, SOS, SMI, grip power, gait speed, and TRACP-5b as independent variables

Selection of modelling was done using stepwise method

${ }^{\mathrm{a}}$ Women $=0$, Men $=1$

formation in immobilized patients [27]. Conversely, sclerostin correlated negatively with TRACP-5b because the bone resorption rate was high in healthy community-dwelling adults in this study.

$\mathrm{OC}$ concentrations were significantly higher in women than in men, and were significantly associated with TRACP-5b. OC concentrations were significantly positively correlated with high force eccentric exercise, which promotes increased bone metabolism [12]. In this study, we investigated the relationship between $\mathrm{OC}$ and muscle parameters, and determined that there was no observable obvious relationship between them.

IGF-1 is known to have an anabolic effect on bone [14]. IGF-1 has been reported to be positively correlated with BMD in elderly women, suggesting a correlation with bone formation [28]. We found that IGF-1 was significantly positively correlated with SOS, suggesting that IGF-1 plays an important role in bone formation. Increased IGF-1 expression with muscle hypertrophy also likely increases IGF-1 secretion and the local abundance of IGF-1 at the muscle-bone interface. Muscle hypertrophy and bone anabolism are connected through an IGF-1 mediated paracrine signaling mechanism [29]. Serum IGF-1 is useful for estimating the prevalence of vertebral fractures in patients with type 2 diabetes mellitus considering that decreased serum IGF-1 may be involved in the deterioration of bone quality [30].

IGF-1 and myostatin, both of which are myokines, mediate crosstalk among myocytes and are thought to be involved in the synthesis and decomposition of muscle proteins [31]. However, no consensus on the involvement of myostatin has been reached at this time. In this 
study, myostatin was not correlated with any of the assessed variables. One of possible reasons for this could be that it is difficult to distinguish between the active and inactive forms of myostatin using conventional quantification methods [18].

Limitations of our study are the relatively small sample size and the cross-sectional study design. The strength of this study is that it is the first to evaluate the association of serum bone- and muscle-derived factors with body composition and physical function simultaneously. In the future, it will be necessary to conduct longitudinal studies to confirm the findings of this study. These studies may eventually help elucidate the relationship between osteoporosis and sarcopenia.

\section{Conclusions}

We found that sclerostin was significantly associated with sex, age, and bone, although there was no discernable relationship between serum sclerostin and muscle function. There was no obvious relationship between $\mathrm{OC}$ and muscle parameters. This study suggests that IGF-1 is an important modulator of not only muscle mass and function but also of bone in communitydwelling middle-aged and elderly adults.

\section{Abbreviations \\ BIA: Bioelectrical impedance analysis; BMD: Bone mineral density; BMI: Body mass index; DXA: Dual energy x-ray absorptiometry; ELISA: Enzyme-linked immuno-sorbent assay; IGF-1: Insulin-like growth factor-1; OC: Osteocalcin; QUS: Quantitative ultrasound; SMI: Skeletal muscle mass index; SOS: Speed of sound; TRACP-5b: Tartrate-resistant acid phosphatase-5b}

\section{Acknowledgments}

The authors sincerely acknowledge the town of Hino in Tottori Prefecture, Japan. The authors also acknowledge all the staff members who were involved in the Good Aging and Intervention Against Nursing Care and Activity Decline (GAINA) study, Hideaki Mitani for his support, and Ryoko Ikehara for her secretarial assistance.

\section{Authors' contributions}

KM designed the study, participated in the study, conducted acquisition, analysis, and interpretation of data, and drafted the manuscript. HM, ST, CT, $\mathrm{MO}$, and $\mathrm{HH}$ helped design the study, participated in the study, and conducted acquisition, analysis, and interpretation of data. HN contributed to the study design and drafted the manuscript. All authors read and approved the final version of the manuscript.

\section{Funding}

This study was supported by a Ministry of Education, Culture, Sports, Science and Technology Grant, a Japanese Society for Musculoskeletal Medicine Grant and Community Contribution Support Project of Tottori University.

\section{Availability of data and materials}

The datasets generated and analyzed during the current study are not publicly available because of professional discretion, as they were part of patient's records, but are available as a de-identified data sheet from the corresponding author on reasonable request.

\section{Ethics approval and consent to participate}

All of the participants provided written informed consent, and the study was approved by the local ethics committee of the Faculty of Medicine Tottori University (No. 2354).
Consent for publication

Not applicable.

\section{Competing interests}

The authors declare that they have no competing interests.

\section{Author details}

${ }^{1}$ Department of Orthopedic Surgery, Faculty of Medicine, Tottori University, Nishicho 36-1, Yonago, Tottori 683-8504, Japan. ${ }^{2}$ Department of Rehabilitation, Faculty of Health Science and Technology, Kawasaki University of Medical Welfare, Matsushima 288, Kurashiki, Okayama 701-0193, Japan. ${ }^{3}$ School of Health Science, Faculty of Medicine, Tottori University, Nishicho 86, Yonago, Tottori 683-8504, Japan. ${ }^{4}$ Rehabilitation Division, Tottori University Hospital, Nishicho 36-1, Yonago, Tottori 683-8504, Japan.

Received: 15 October 2018 Accepted: 22 May 2019

Published online: 05 June 2019

\section{References}

1. Lewiecki EM, Watts NB. New guidelines for the prevention and treatment of osteoporosis. South Med J. 2009;102:175-9.

2. Matsumoto $\mathrm{H}$, Tanimura C, Tanishima S, Osaki M, Noma H, Hagino H. Sarcopenia is a risk factor for falling in independently living Japanese older adults: a 2-year prospective cohort study of the GAINA study. Geriatr Gerontol Int. 2017;17:2124-30.

3. Miyakoshi N, Hongo M, Mizutani Y, Shimada Y. Prevalence of sarcopenia in Japanese women with osteopenia and osteoporosis. J Bone Miner Metab. 2013;31:556-61.

4. Wu CH, Yang KC, Chang HH, Yen JF, Tsai KS, Huang KC. Sarcopenia is related to increased risk for low bone mineral density. J Clin Densitom. 2013:16:98-103.

5. Morse A, McDonald MM, Kelly NH, Melville KM, Schindeler A, Kramer I, Kneissel M, van der Meulen MC, Little DG. Mechanical load increases in bone formation via a sclerostin-independent pathway. J Bone Miner Res. 2014;29:2456-67.

6. Ke HZ, Richards WG, Li X, Ominsky MS. Sclerostin and Dickkopf-1 as therapeutic targets in bone diseases. Endocr Rev. 2012;33:747-83.

7. Amrein K, Amrein S, Drexler C, Dimai HP, Dobnig H, Pfeifer K, Tomaschitz A, Pieber TR, Fahrleitner-Pammer A. Sclerostin and its association with physical activity, age, gender, body composition, and bone mineral content in healthy adults. J Clin Endocrinol Metab. 2012;97:148-54.

8. Szulc P, Boutroy S, Vilayphiou N, Schoppet M, Rauner M, Chapurlat R, Hamann C, Hofbauer LC. Correlates of bone microarchitectural parameters and serum sclerostin levels in men: the STRAMBO study. J Bone Miner Res. 2013;28:1760-70.

9. Garnero P, Sornay-Rendu E, Munoz F, Borel O, Chapurlat RD. Association of serum sclerostin with bone mineral density, bone turnover, steroid and parathyroid hormones, and fracture risk in postmenopausal women: the OFELY study. Osteoporos Int. 2013;24:489-94.

10. Invernizzi M, Carda S, Rizzi M, Grana E, Squarzanti DF, Cisari C, Molinari C, Reno F. Evaluation of serum myostatin and sclerostin levels in chronic spinal cord injured patients. Spinal Cord. 2015;53:615-20.

11. Lee AJ, Hodges S, Eastell R. Measurement of osteocalcin. Ann Clin Biochem. 2000;37 ( Pt 4:432-46.

12. Tsuchiya $Y$, Sakuraba K, Ochi E. High force eccentric exercise enhances serum tartrate-resistant acid phosphatase-5b and osteocalcin. J Musculoskelet Neuronal Interact. 2014;14:50-7.

13. Kaji H. Effects of myokines on bone. Bonekey Rep. 2016;5:826

14. Lombardi G, Di Somma C, Vuolo L, Guerra E, Scarano E, Colao A. Role of IGF-I on PTH effects on bone. J Endocrinol Investig. 2010;33:22-6.

15. Barbieri M, Ferrucci L, Ragno E, Corsi A, Bandinelli S, Bonafe M, Olivieri F, Giovagnetti S, Franceschi C, Guralnik JM, Paolisso G. Chronic inflammation and the effect of IGF-I on muscle strength and power in older persons. Am J Physiol Endocrinol Metab. 2003;284:E481-7.

16. Hofmann M, Halper B, Oesen S, Franzke B, Stuparits P, Tschan H, Bachl N, Strasser EM, Quittan M, Ploder M, Wagner KH, Wessner B. Serum concentrations of insulin-like growth factor-1, members of the TGF-beta superfamily and follistatin do not reflect different stages of dynapenia and sarcopenia in elderly women. Exp Gerontol. 2015;64:35-45.

17. Volpato S, Bianchi L, Cherubini A, Landi F, Maggio M, Savino E, Bandinelli S, Ceda GP, Guralnik JM, Zuliani G, Ferrucci L. Prevalence and clinical correlates 
of sarcopenia in community-dwelling older people: application of the EWGSOP definition and diagnostic algorithm. J Gerontol A Biol Sci Med Sci. 2014;69:438-46.

18. White TA, LeBrasseur NK. Myostatin and sarcopenia: opportunities and challenges - a mini-review. Gerontology. 2014;60:289-93.

19. Yarasheski KE, Bhasin S, Sinha-Hikim I, Pak-Loduca J, Gonzalez-Cadavid NF. Serum myostatin-immunoreactive protein is increased in 60-92 year old women and men with muscle wasting. J Nutr Health Aging. 2002;6:343-8.

20. Burch PM, Pogoryelova O, Palandra J, Goldstein R, Bennett D, Fitz L, Guglieri M, Bettolo CM, Straub V, Evangelista T, Neubert H, Lochmuller H, Morris C. Reduced serum myostatin concentrations associated with genetic muscle disease progression. J Neurol. 2017;264:541-53.

21. Bergen HR 3rd, Farr JN, Vanderboom PM, Atkinson EJ, White TA, Singh RJ, Khosla S, LeBrasseur NK. Myostatin as a mediator of sarcopenia versus homeostatic regulator of muscle mass: insights using a new mass spectrometry-based assay. Skelet Muscle. 2015;5:21.

22. Morissette MR, Stricker JC, Rosenberg MA, Buranasombati C, Levitan EB, Mittleman MA, Rosenzweig A. Effects of myostatin deletion in aging mice. Aging Cell. 2009;8:573-83.

23. Matsumoto H, Hagino H, Osaki M, Tanishima S, Tanimura C, Matsuura A, Makabe T. Gait variability analysed using an accelerometer is associated with locomotive syndrome among the general elderly population: the GAINA study. J Orthop Sci. 2016;21:354-60.

24. Tanimura C, Matsumoto H, Tokushima Y, Yoshimura J, Tanishima S, Hagino H. Self-care agency, lifestyle, and physical condition predict future frailty in community-dwelling older people. Nurs Health Sci. 2017.

25. Tanishima S, Hagino H, Matsumoto $H$, Tanimura C, Nagashima $H$. Association between sarcopenia and low back pain in local residents prospective cohort study from the GAINA study. BMC Musculoskelet Disord. 2017;18:452.

26. Coulson J, Bagley L, Barnouin Y, Bradburn S, Butler-Browne G, Gapeyeva H, Hogrel JY, Maden-Wilkinson T, Maier AB, Meskers C, Murgatroyd C, Narici M, Paasuke M, Sassano L, Sipila S, Al-Shanti N, Stenroth L, Jones DA, McPhee JS, Circulating levels of dickkopf-1, osteoprotegerin and sclerostin are higher in old compared with young men and women and positively associated with whole-body bone mineral density in older adults. Osteoporos Int. 2017.

27. Gaudio A, Pennisi P, Bratengeier C, Torrisi V, Lindner B, Mangiafico RA, Pulvirenti I, Hawa G, Tringali G, Fiore CE. Increased sclerostin serum levels associated with bone formation and resorption markers in patients with immobilization-induced bone loss. J Clin Endocrinol Metab. 2010;95:2248-53.

28. Hamrick MW, McNeil PL, Patterson SL. Role of muscle-derived growth factors in bone formation. J Musculoskelet Neuronal Interact. 2010;10:64-70.

29. Hamrick MW. A role for myokines in muscle-bone interactions. Exerc Sport Sci Rev. 2011;39:43-7.

30. Kanazawa I, Notsu M, Miyake H, Tanaka K, Sugimoto T. Assessment using serum insulin-like growth factor-l and bone mineral density is useful for detecting prevalent vertebral fractures in patients with type 2 diabetes mellitus. Osteoporos Int. 2018.

31. Hitachi K, Nakatani M, Tsuchida K. Myostatin signaling regulates Akt activity via the regulation of miR-486 expression. Int J Biochem Cell Biol. 2014;47: 93-103.

\section{Publisher's Note}

Springer Nature remains neutral with regard to jurisdictional claims in published maps and institutional affiliations.

Ready to submit your research? Choose BMC and benefit from:

- fast, convenient online submission

- thorough peer review by experienced researchers in your field

- rapid publication on acceptance

- support for research data, including large and complex data types

- gold Open Access which fosters wider collaboration and increased citations

- maximum visibility for your research: over $100 \mathrm{M}$ website views per year

At BMC, research is always in progress.

Learn more biomedcentral.com/submissions 